\title{
Use of Both Serum Cystatin C and Creatinine as Diagnostic Criteria for Cardiac Surgery- Associated Acute Kidney Injury and Its Correlation with Long-Term Major Adverse Events
}

\author{
Miaolin Che ${ }^{a}$ Xudong Wang ${ }^{b}$ Bo Xie ${ }^{b}$ Ritai Huang ${ }^{b}$ Shang Liu ${ }^{a}$ \\ Yucheng Yan ${ }^{a}$ Mingli Zhu ${ }^{a}$ Renhua Lu $^{a}$ Jiaqi Qian ${ }^{a}$ Weiming Zhang ${ }^{\text {a }}$ \\ Leyi Gu ${ }^{a}$ Shan $\mathrm{Mou}^{a}$ Zhaohui $\mathrm{Ni}^{\mathrm{a}}$ Song Xue ${ }^{\mathrm{b}}$ \\ a Department of Nephrology, Renji Hospital, School of Medicine, Shanghai Jiao Tong \\ University, Shanghai, China; ${ }^{b}$ Department of Cardiovascular Surgery, Renji Hospital, School \\ of Medicine, Shanghai Jiao Tong University, Shanghai, China
}

\author{
Keywords \\ Cardiac surgery · Acute kidney injury · Diagnosis · Prognosis · Risk stratification
}

\begin{abstract}
Background/Aims: Cardiac surgery-associated acute kidney injury (CSA-AKI) was traditionally defined as an increase in serum creatinine $(\mathrm{s} C r)$ after cardiac surgery. Recently, serum cystatin $\mathrm{C}(\mathrm{s} C \mathrm{C} C)$ has been proposed to be a better biomarker in the prediction of AKI. The clinical utility and performance of combining $\mathrm{sCyC}$ and $\mathrm{sCr}$ in patients with $\mathrm{AKI}$, particularly for the prediction of long-term outcomes, remain unknown. Methods: We measured sCyC together with sCr in 628 patients undergoing cardiac surgery. $\mathrm{sCyC}$ and $\mathrm{s} C r$ were assessed at baseline and 24 and $48 \mathrm{~h}$ after surgery. CSA-AKI determined by $\mathrm{sCr}\left(\mathrm{CSA}-\mathrm{AKI}_{\mathrm{s} \mathrm{Cr}}\right)$ was defined as an $\mathrm{sCr}$ increase greater than $0.3 \mathrm{mg} / \mathrm{dL}$ or $50 \%$ from baseline. Major adverse events (MAEs; including death of any cause and dialysis) at 3 years were assessed. Results: $\mathrm{CSA}-\mathrm{AKI}_{\mathrm{s}} \mathrm{Cr}$ developed in 178 patients (28.3\%). Three-year follow-up was available for 621 patients; MAEs occurred in 42 patients (6.8\%). An increase in $\mathrm{sCyC}$ concentration $\geq 30 \%$ within $48 \mathrm{~h}$ after surgery was detected in 228 patients (36.3\%). This was the best sCyC cutoff for CSA-AKI ${ }_{\mathrm{sCr}}$ detection (negative predictive value $=88.8 \%$, positive predictive value $=58.3 \%$ ). To evaluate the use of both sCyC and sCr as CSA-AKI diagnostic criteria, we stratified patients into 3 groups: non-CSA-AKI, CSA-AKI detected by a single marker, and CSA-AKI detected by both markers. By multivariable logistic regression analysis, the independent predictors of MAEs at 3 years
\end{abstract}

M.C. and X.W. are co-first authors and contributed equally.

Zhaohui Ni

Department of Nephrology, Renji Hospital

School of Medicine, Shanghai Jiao Tong University

Shanghai 200127 (China)

E-Mail drnizhaohui@126.com
Song Xue Department of Cardiovascular Surgery

Renji Hospital School of Medicine

Shanghai Jiao Tong University

Shanghai 200127 (China)

E-Mail renjixuesong@gmail.com 
were group 2 (non-CSA-AKI group as the reference, CSA-AKI detected by a single marker: odds ratio $[O R]=3.48,95 \%$ confidence interval $[\mathrm{Cl}]: 1.27-9.58, p=0.016)$, group 3 (CSA-AKI detected by both markers: $\mathrm{OR}=5.12,95 \% \mathrm{Cl}: 2.01-13.09 ; p=0.001)$, and baseline glomerular filtration rate (OR $=2.24 ; 95 \% \mathrm{Cl}: 1.27-3.95 ; p=0.005)$. Conclusion: Combining $\mathrm{sCyC}$ and $\mathrm{sCr}$ to diagnose CSA-AKI would be beneficial for risk stratification and prognosis in patients after cardiac surgery.

(C) 2019 The Author(s)

Published by S. Karger AG, Basel

\section{Background}

Acute kidney injury (AKI) is the most common major complication of cardiac surgery and is associated with high mortality and morbidity rates [1-3]. Serum creatinine ( $\mathrm{sCr}$ ) remains the clinical standard for AKI diagnosis, and increases of $0.3 \mathrm{mg} / \mathrm{dL}$ or higher are independently associated with a longer hospital stay and both in-hospital and long-term death [4-6]. However, its usefulness may not be ideal in the postoperative setting due to hemodilution [7] and blood loss [8]. Furthermore, decreases in creatinine generation from muscle [9] may occur in the perioperative setting. Thus, $\mathrm{sCr}$ is known as an insensitive and nonspecific parameter for renal function evaluation.

Responding to the need for early and sensitive identification of patients with AKI, several biomarkers have been proposed over the past few years [10-13]. Perhaps most well-studied is cystatin $\mathrm{C}$, a 13-kDa cysteine protease inhibitor that is produced by all nucleated cells at a constant rate and is renally cleared. Therefore, serum cystatin $\mathrm{C}(\mathrm{sCyC})$ level is determined by the glomerular filtration rate [14]. Based on its physiological metabolism characteristics, the life cycle of sCyC is merely half of that of sCr (1.5-2 vs. $4 \mathrm{~h}$ ). Namely, once renal function is fluctuating, $\mathrm{sCyC}$ changes much earlier than $\mathrm{sCr}[15,16]$. Recently, $\mathrm{sCyC}$ has been proposed as a superior marker to $\mathrm{sCr}$ for detection of early changes in GFR and as a marker of acute injury to the kidney $[17,18]$. Besides, $\mathrm{sCyC}$ can be assayed easily, and routine laboratory measurement increasingly is becoming available to clinicians. However, use of sCyC level for cardiac surgery-associated AKI (CSA-AKI) diagnosis remains controversial, and limited data exist on whether changes in the composite of $\mathrm{sCyC}$ and $\mathrm{sCr}$ are superior to $\mathrm{sCr}$ in predicting future major adverse events (MAEs) following cardiac surgery [19].

In the present study, we performed a prospective study comparing changes in $\mathrm{sCr}$ and sCyC in patients at risk for CSA-AKI following cardiac surgery. The main purposes of this study were to (1) assess the optimal sCyC cutoff point to detect CSA-AKI; (2) determine whether the use of both sCyC and sCr would help to capture associations of in-hospital changes in renal function with prognosis after hospital discharge.

\section{Methods}

\section{Design and Participants}

Between July 2011 and March 2013, patients over 18 years of age who were scheduled to undergo cardiac surgery (including coronary artery bypass grafting [CABG], valve surgery, combined CABG and valve procedures, and other cardiac surgeries such as congenital heart disease repair, aorta aneurysm, others) at Renji Hospital, Shanghai Jiao Tong University School of Medicine, Shanghai, China were prospectively recruited. Exclusion criteria include patients under 18 years of age, patients with pre-existing end-stage kidney disease, patients receiving any form of dialysis therapy before surgery, and patients who had died during hospitalization. 
Table 1. New definition of CSA-AKI using both sCr and sCyC

\begin{tabular}{lll}
\hline Group & Definition & Risk stratification \\
\hline Group 1 & $\begin{array}{l}\text { Non-CSA-AKI: sCr increase }<0.3 \mathrm{mg} / \mathrm{dL} \text { and } 50 \% \text { from baseline; } \\
\text { and sCyC increase }<30 \% \text { from baseline }\end{array}$ & No risk \\
\hline Group 2 & $\begin{array}{l}\text { CSA-AKI detected by a single marker: fulfilling only } 1 \text { of the criteria } \\
\text { below: }(1) \mathrm{sCr} \text { increase }>0.3 \mathrm{mg} / \mathrm{dL} \text { or } 50 \% \text { from baseline; }(2) \mathrm{sCyC} \\
\text { increase } \geq 30 \% \text { from baseline }\end{array}$ & Potential risk \\
\hline Group 3 & $\begin{array}{l}\text { CSA-AKI detected by both markers: } \mathrm{SCr} \text { increase }>0.3 \mathrm{mg} / \mathrm{dL} \\
\text { or } 50 \% \text { from baseline; and sCyC increase } \geq 30 \% \text { from baseline }\end{array}$ & High risk \\
\hline
\end{tabular}

CSA-AKI, cardiac surgery-associated acute kidney injury; sCr, serum creatinine; $\mathrm{sCyC}$, serum cystatin C.

Data Collection, Biomarker Measurement, and Follow-Up

Prespecified demographic and clinical data were recorded for each participant. Blood samples for biomarker measurement were collected at baseline and postsurgery day 1 and day 2 (at the time of routine morning blood collection). Blood samples were collected, centrifuged at $2,000 \mathrm{~g}$ for $5 \mathrm{~min}$, and the supernatants aliquoted and stored at $-80^{\circ} \mathrm{C}$. sCyC was measured by immunonephelometry (Dade Behring, Marburg, Germany) using a fully automatic chemistry analyzer (Hitachi 7180). sCr was measured by a modified Jaffe method with protein precipitation using an alkaline picrate reaction. $\mathrm{sCr}$ and $\mathrm{sCyC}$ were measured in the central biochemistry laboratory of Renji Hospital. All the participants were scheduled to follow-up until 3 years postsurgery by outpatient clinic visit or telephone interview. Reported events were carefully evaluated and recorded.

\section{Definitions and Risk Factors}

CSA-AKI as determined by $\mathrm{sCr}\left(\mathrm{CSA}-\mathrm{AKI}_{\mathrm{sCr}}\right)$ was defined as an increase greater than 0.3 $\mathrm{mg} / \mathrm{dL}$ or $50 \%$ in $\mathrm{sCr}$ from baseline within $48 \mathrm{~h}$ after surgery [20], which differs from the Kidney Disease: Improving Global Outcomes (KDIGO) definition by not incorporating oliguria

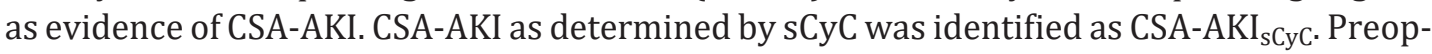
erative variables and surgery types known to be or which could potentially be associated with AKI or other adverse outcomes were collected. These included age, gender, hypertension (SBP $\geq 140 \mathrm{~mm} \mathrm{Hg}$ and/or DBP $\geq 90 \mathrm{~mm} \mathrm{Hg}$ ), diabetes mellitus (using oral hypoglycemic agents or insulin), peripheral artery disease (determined by clinical diagnosis or imaging results), cerebrovascular disease (previous cerebral vascular accident such as ischemic or hemorrhagic), NY Heart Association (NYHA) functional class III or IV, chronic obstructive pulmonary disease (COPD), recent myocardial infarction (MI; i.e., $<30$ days before surgery), and hyperuricemia (a serum uric acid $>6.6$ in women and $>7.0 \mathrm{mg} / \mathrm{dL}$ in men), left ventricular ejection fraction (LVEF) (assessed preoperatively by echocardiography), and proteinuria (urine dipstick test result of $1+$ or greater). The eGFR was calculated with the MDRD4 equation. The Cleveland Clinic Score was calculated according to the algorithm reported by Thakar et al. [21]. MAEs were defined as death from any cause and further deterioration of renal function requiring chronic dialysis.

\section{Statistical Analyses}

Continuous variables were expressed as mean \pm SD and analyzed by unpaired Student's $t$ test. Non-parametric variables were expressed as median and $25-75 \%$ percentiles and analyzed by Mann-Whitney's test. Categorical variables were expressed as absolute $(n)$ and 
Kidney

Blood Pressure

Research

Table 2. Clinical and biochemical characteristics of patients undergoing cardiac surgery $(n=$ 628)

\begin{tabular}{l|l}
\hline Kidney Blood Press Res 2019;44:415-425 \\
\hline DOI: 10.1159/000499647 & $\begin{array}{l}\text { (c) 2019 The Author(s). Published by S. Karger AG, Basel } \\
\text { www.karger.com/kbr }\end{array}$ \\
\hline
\end{tabular}

Che et al.: Use of Both sCyC and sCr as Diagnostic Criteria for CSA-AKI

\section{Variables}

Clinical variables

Age, years

Male

$60.5 \pm 13.1[18-81]$

Diabetes mellitus

$403(64.2)$

Hypertension

Peripheral artery disease

$115(18.3)$

Cerebrovascular disease

$201(32.0)$

$11(1.8)$

COPD

$24(3.8)$

$10(1.6)$

$58(9.2)$

LVEF $<45 \%$

$113(18.0)$

NYHA grade III-IV

$28(4.5)$

Previous cardiac surgery

$58(9.2)$

Recent MI

$222(35.4)$

Hyperuricemia

$146(23.2)$

Proteinuria

$36(5.7)$

Use of statins

Type of surgery

CABG

Valve

CABG + valve

$246(39.2)$

CHD

$289(46.0)$

$58(9.2)$

$5(0.8)$

Aortic

19 (3.0)

Others

$11(1.8)$

CPB

455 (72.5)

CPB time, min

$101.0[78.5-134.0]$

\section{Biochemical variables}

sCr, mg/dL

Baseline

24-48 h postsurgery

sCyC, mg/dL

Baseline

24-48 h postsurgery

Baseline eGFR, $\mathrm{mL} / \mathrm{min}$ per $1.73 \mathrm{~m}^{2}$

$\geq 90$

60-89

$<60$

0.81 [0.66-0.98]

$1.01[0.80-1.30]^{*}$

$1.02[0.96-1.14]$

$1.14[0.94-1.47]^{\#}$

$94.1[75.6-117.8]$

355 (56.5)

231 (36.8)

42 (6.7)

Cleveland Clinic Score
0-2
349 (55.6)
3-4
$216(34.4)$
$\geq 5$
$63(10.0)$

Continuous values are expressed as median and interquartile range [IQR] (sCr and $\mathrm{CyC}$ ); categorical values are expressed as number (percentage). COPD, chronic obstructive pulmonary disease; LVEF, left ventricular ejection fraction; NYHA, New York Heart Association; MI, myocardial infarction; $\mathrm{CPB}$, cardiopulmonary bypass; CABG, coronary artery bypass grafting; CHD, congenital heart disease; $\mathrm{sCr}$, serum creatinine; sCyC, serum cystatin C; eGFR, estimated glomerular filtration rate; CSA-AKI, cardiac surgery-associated acute kidney injury. ${ }^{*} p<0.05$, compared with $\mathrm{sCr}$ at baseline; ${ }^{\#} p<0.05$, compared with sCyC at baseline. 


\section{Kidney \\ Blood Pressure \\ Research}

Table 3. Occurrence of MAEs at 3 -year follow-up $(n=621)$

\begin{tabular}{l|l}
\hline Kidney Blood Press Res 2019;44:415-425 \\
\hline DOI: 10.1159/000499647 & $\begin{array}{l}\text { ○ 2019 The Author(s). Published by S. Karger AG, Basel } \\
\text { www.karger.com/kbr }\end{array}$ \\
\hline
\end{tabular}

Che et al.: Use of Both sCyC and sCr as Diagnostic Criteria for CSA-AKI

\begin{tabular}{lr}
\hline Event & $n(\%)$ \\
\hline All-cause death & $41(6.6)$ \\
Cardiac death & $21(3.4)$ \\
Pulmonary infection & $12(1.9)$ \\
Stroke & $7(1.1)$ \\
Tumor & $1(0.2)$ \\
Nephropathy requiring chronic dialysis & $1(0.2)$ \\
MAEs & $42(6.8)$ \\
\hline
\end{tabular}

MAEs, major adverse events.

relative (\%) frequency and were analyzed by Chi-squared analysis, as appropriate. The diagnostic accuracy of the $\mathrm{SCyC}$ increment above baseline for predicting CSA-AKI as determined by sCr was evaluated by receiver operating characteristic (ROC) curve analysis. Subsequently, from the ROC analysis, a $\mathrm{SCyC}$ cutoff increment value was chosen on the basis of maximum sensitivity and specificity. Whether the change in $\mathrm{sCyC}$ was an independent predictor of MAEs was determined by multivariable logistic regression analysis using Firth's penalized-likelihood estimation as the number of 3-year MAEs was relative small [22]. Interaction effects between an sCyC increase greater than $30 \%$ and other covariates were assessed. Finally, a new definition of CSA-AKI (CSA-AKI $\mathrm{New}_{\text {) }}$ ) was proposed using both $\mathrm{sCr}$ and sCyC (Table 1). By this definition, participants were further stratified into 3 groups as follows: non-CSA-AKI, CSA-AKI detected only by a single marker, and CSA-AKI detected by both markers. Baseline characters, Cleveland Clinic Score, and MAEs incidence were compared across the 3 groups. $\mathrm{CSA}-\mathrm{AKI}_{\mathrm{New}}$ was included as a covariate in the multivariable logistic regression model using Firth's penalized-likelihood estimation to assess whether the new definition of CSA-AKI was an independent predictor of MAEs, along with age, diabetes mellitus, recent MI, NYHA grade, baseline eGFR, and Cleveland Clinic Score. If statistically significant, the interaction term was included into the regression model. $p<0.05$ was considered significant throughout the analyses. All analyses were performed using SPSS 20.0 and R 3.3.2 software.

\section{Results}

\section{Demographic Characteristics}

A total of 628 participants were included in the study. Baseline characteristics of participants are listed in Table 2. Median sCr concentration in these participants significantly

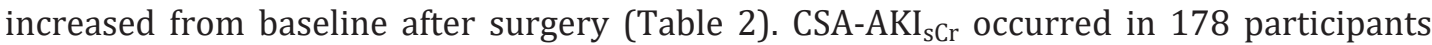
(28.3\%). Three-year follow-up information was available for 621 participants (98.9\%). MAEs occurred in 42 participants (6.8\%, Table 3). In particular, death occurred in 41 patients $(6.6 \%)$ and chronic dialysis in 1 patient $(0.2 \%)$.

\section{Serum Cystatin C and Predictive Value for Clinical Outcomes}

Median sCyC concentration significantly changed from baseline after surgery (Table 2). The distributions in sCyC level changes after surgery are presented in Table 4. By ROC curve analysis, the change in $\mathrm{SCyC}$ from baseline significantly predicted the development of CSA$\mathrm{AKI}_{\mathrm{sCr}}$ (area under the curve $[\mathrm{AUC}]=0.843,95 \% \mathrm{CI}=0.809-0.877, p<0.001$ ) (Fig. 1). To optimize both sensitivity and specificity, an sCyC increase greater than $30 \%$ after surgery, 
Table 4. Distribution of $\mathrm{sCyC}$ level changes after cardiac surgery and relationship with $\mathrm{CSA}-\mathrm{AKI}_{\mathrm{sCr}}$

\begin{tabular}{lllllll}
\hline $\begin{array}{l}\text { Changes } \\
\text { in sCyC }\end{array}$ & $\begin{array}{l}\text { Proportion, } \\
n(\%)\end{array}$ & $\begin{array}{l}\text { Sensitivity, } \\
\%\end{array}$ & $\begin{array}{l}\text { Specificity, } \\
\%\end{array}$ & $\begin{array}{l}\text { PPV, } \\
\%\end{array}$ & $\begin{array}{l}\text { NPV, } \\
\%\end{array}$ & $\begin{array}{l}\text { Youden } \\
\text { index }\end{array}$ \\
\hline$\geq 20 \%$ & $293(46.7)$ & 80 & 68 & 48.8 & 89.6 & 0.49 \\
$\geq 25 \%$ & $256(40.8)$ & 77 & 75 & 53.5 & 89.0 & 0.52 \\
$\geq 30 \%$ & $228(36.3)$ & 75 & 80 & 58.3 & 88.8 & 0.55 \\
$\geq 35 \%$ & $199(31.7)$ & 69 & 84 & 62.3 & 87.4 & 0.53 \\
\hline
\end{tabular}

sCyC, serum cystatin C; PPV, positive predictive value; NPV, negative predictive value.

Fig. 1. ROC curve and AUC showing the diagnostic performance of $\mathrm{sCyC}$ for CSA-AKI detection. $\mathrm{AUC}=$ $0.843(p<0.001)$.

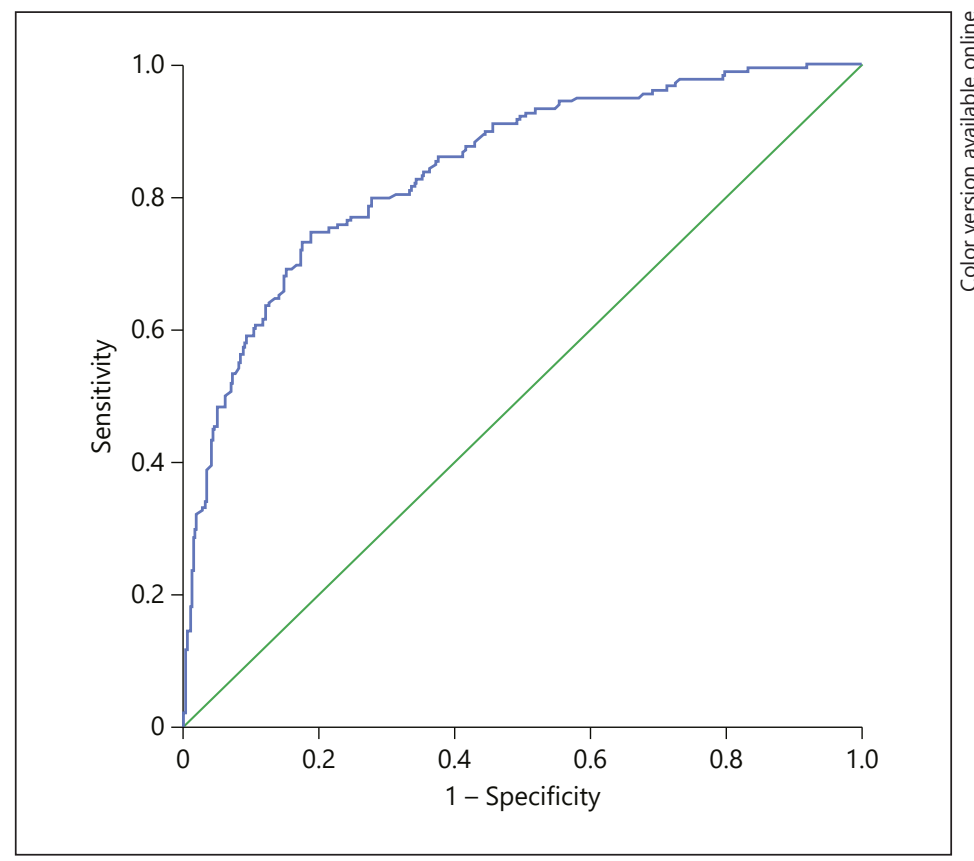

which had an $75 \%$ sensitivity and $80 \%$ specificity, was chosen as the optimal cutoff value (Table 4). Using this definition, participants who developed CSA-AKI ${ }_{\mathrm{SCyC}}$ had higher Cleveland Clinic Scores and experienced more MAEs compared with participants without CSA-AKI $\mathrm{SCyC}_{\mathrm{C}}$ (Fig. 2). Multivariable logistic regression analysis revealed that an $\mathrm{SCyC}$ increase greater than $30 \%$ was an independent predictor of 3-year MAEs (adjusted OR $=2.98,95 \%$ CI: 1.41-6.30, $p=0.004)$.

\section{Association of the New Definition of CSA-AKI with Long-Term MAEs}

Based on the new definition of CSA-AKI listed in Table 1, participants were further stratified into 3 groups. The baseline characteristics revealed the risk of developing CSA-AKI increased across the 3 groups (Table 5). Participants in the non-CSA-AKI group had the lowest Cleveland Clinic Scores, and participants in the group with CSA-AKI detected by both markers had the highest Cleveland Clinic Scores (Fig. 3). A similar pattern was observed in the occurrence of MAEs. The occurrence of MAEs was lowest in the non-CSA-AKI group and the highest in participants with CSA-AKI detected by both markers (Fig. 3). By multivariable logistic regression analysis, a significant correlation was found between MAEs and the new definition 
Table 5. Comparisons of clinical characteristics in patients stratified by composite of sCr and sCyC $(n=628)$

\begin{tabular}{|c|c|c|c|c|}
\hline Variables & $\begin{array}{l}\text { Non-CSA-AKI } \\
(n=355)\end{array}$ & $\begin{array}{l}\text { CSA-AKI detected } \\
\text { by a single marker } \\
(n=140)\end{array}$ & $\begin{array}{l}\text { CSA-AKI detected } \\
\text { by both markers } \\
(n=133)\end{array}$ & $p$ value \\
\hline \multicolumn{5}{|l|}{ Clinical variables } \\
\hline Age, years & $58.2 \pm 13.2$ & $61.2 \pm 13.0$ & $65.8 \pm 11.3$ & $<0.001$ \\
\hline Male & $223(62.8)$ & $91(65.0)$ & $89(66.9)$ & 0.683 \\
\hline Diabetes mellitus & $61(17.2)$ & $20(14.3)$ & $34(25.6)$ & 0.039 \\
\hline Hypertension & $104(29.3)$ & $36(25.7)$ & $61(45.9)$ & $<0.001$ \\
\hline Peripheral artery disease & $2(0.6)$ & $4(2.9)$ & $5(3.8)$ & 0.030 \\
\hline Cerebrovascular disease & $10(2.8)$ & $8(5.7)$ & $6(4.5)$ & 0.302 \\
\hline COPD & $5(1.4)$ & $2(1.4)$ & $3(2.3)$ & 0.789 \\
\hline Hyperuricemia & $67(18.9)$ & $35(25.0)$ & $44(33.1)$ & 0.004 \\
\hline Previous cardiac surgery & $10(2.8)$ & 8 (5.7) & $10(7.5)$ & 0.056 \\
\hline Recent MI & $30(8.5)$ & $13(9.3)$ & $15(11.3)$ & 0.506 \\
\hline NYHA grade III-IV & $45(12.7)$ & $26(18.6)$ & $42(31.6)$ & $<0.001$ \\
\hline LVEF $<45 \%$ & $26(7.3)$ & $16(11.4)$ & $16(12.0)$ & 0.183 \\
\hline Preoperative use of statins & $140(39.4)$ & $50(35.7)$ & $58(43.6)$ & 0.411 \\
\hline Anemia & $106(29.9)$ & $52(37.1)$ & $64(48.1)$ & 0.001 \\
\hline Proteinuria & $13(3.7)$ & $6(4.3)$ & $17(12.8)$ & $<0.001$ \\
\hline Type of surgery & & & & $<0.001$ \\
\hline CABG & $148(41.7)$ & $51(36.4)$ & $47(35.3)$ & \\
\hline Valve & $175(49.3)$ & $68(48.6)$ & $46(34.6)$ & \\
\hline $\mathrm{CABG}+$ valve & $17(4.8)$ & $14(10.0)$ & $27(20.3)$ & \\
\hline CHD & $3(0.8)$ & $1(0.7)$ & $1(0.8)$ & \\
\hline Aortic & $7(2.0)$ & $3(2.1)$ & $9(6.8)$ & \\
\hline Others & $5(1.4)$ & $3(2.1)$ & $3(2.3)$ & \\
\hline СРB & $248(69.9)$ & $100(71.4)$ & $107(80.5)$ & 0.063 \\
\hline CPB time, min & 95 [71-127] & $100[80-128]$ & $128[93-161]$ & $<0.001$ \\
\hline \multicolumn{5}{|l|}{ Biochemical variables } \\
\hline \multicolumn{4}{|l|}{ Baseline eGFR, mL/min per $1.73 \mathrm{~m}^{2}$} & $<0.001$ \\
\hline $\begin{array}{l}\geq 90 \\
60-89\end{array}$ & $\begin{array}{l}234(65.9) \\
108(30.4)\end{array}$ & $\begin{array}{l}70(50.0) \\
59(42.1)\end{array}$ & $\begin{array}{l}51(38.3) \\
64(48.1)\end{array}$ & \\
\hline$<60$ & $13(3.7)$ & $11(7.9)$ & $18(13.5)$ & \\
\hline \multicolumn{4}{|l|}{ Cleveland Clinic Score } & $<0.001$ \\
\hline $0-2$ & $221(62.3)$ & $84(60.0)$ & $44(33.1)$ & \\
\hline $3-4$ & $117(33.0)$ & $37(26.4)$ & $62(46.6)$ & \\
\hline$\geq 5$ & $17(4.8)$ & $19(13.6)$ & $27(20.3)$ & \\
\hline \multicolumn{5}{|l|}{$\mathrm{sCr}, \mathrm{mg} / \mathrm{dL}$} \\
\hline Baseline & 0.78 [0.64-0.92] & 0.85 [0.68-1.01] & 0.88 [0.72-1.08] & $<0.001$ \\
\hline After $24 \mathrm{~h}$ & $0.80[0.67-1.00]$ & $0.94[0.79-1.16]$ & 1.35 [1.06-1.63] & $<0.001$ \\
\hline After $48 \mathrm{~h}$ & $0.75[0.61-0.91]$ & $0.92[0.74-1.12]$ & $1.42[1.15-1.94]$ & $<0.001$ \\
\hline \multicolumn{5}{|l|}{ sCyC, mg/dL } \\
\hline Baseline & $1.00[0.96-1.04]$ & $1.00[0.82-1.04]$ & 1.02 [1.01-1.06] & 0.030 \\
\hline After $24 \mathrm{~h}$ & 0.92 [0.76-1.07] & 1.14 [0.97-1.34] & 1.56 [1.22-1.93] & $<0.001$ \\
\hline After $48 \mathrm{~h}$ & 0.97 [0.91-1.10] & 1.31 [1.15-1.64] & $1.73[1.36-2.12]$ & $<0.001$ \\
\hline
\end{tabular}

Continuous values are expressed as median and interquartile range [IQR] (sCr and sCyC); categorical values are expressed as number (percentage). COPD, chronic obstructive pulmonary disease; LVEF, left ventricular ejection fraction; NYHA, New York Heart Association; MI, myocardial infarction; CPB, cardiopulmonary bypass; CABG, coronary artery bypass grafting; CHD, congenital heart disease; sCr, serum creatinine; sCyC, serum cystatin C; eGFR, estimated glomerular filtration rate; CSA-AKI, cardiac surgeryassociated acute kidney injury. 


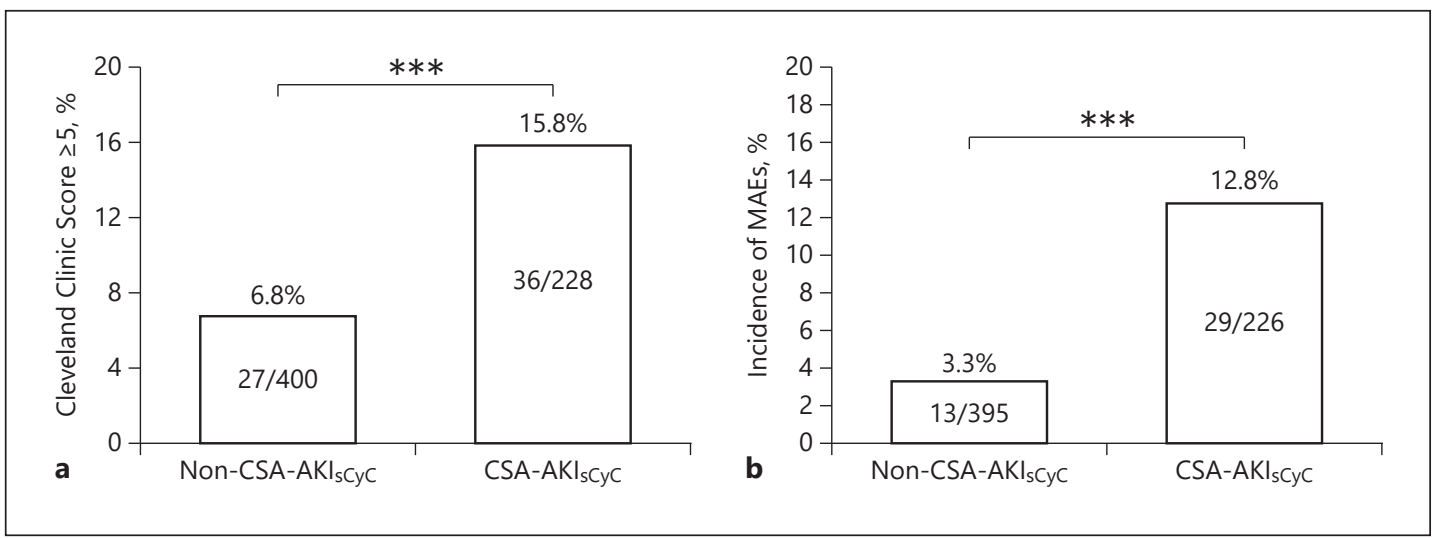

Fig. 2. Portion of patients with Cleveland Clinic Score $\geq 5$ (a) and incidence of MAEs (b) in the CSA-AKI $I_{s C y C}$ group and the non-CSA-AKI $\mathrm{sCyC}_{\mathrm{C}}$ group. ${ }^{* * *} p<0.01$.

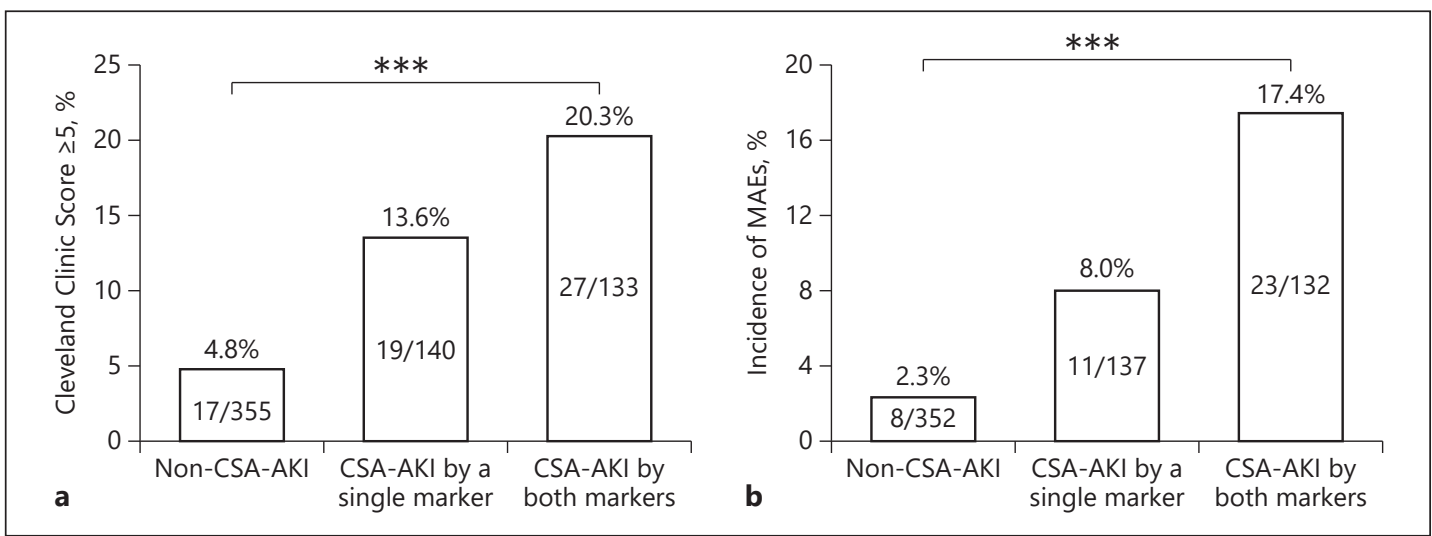

Fig. 3. Portion of patients with Cleveland Clinic Score $\geq 5$ (a) and incidence of MAEs (b) in patients stratified by the composite of sCyC and sCR. ${ }^{* * *} p<0.001$.

of CSA-AKI. Taking the non-CSA-AKI group as the reference, CSA-AKI detected by a single marker (adjusted OR $=3.48,95 \% \mathrm{CI}: 1.27-9.59, p=0.016$ ) and CSA-AKI detected by both markers (adjusted OR $=5.12,95 \% \mathrm{CI}: 2.01-13.09 ; p=0.001$ ) were independent predictors of MAEs at 3 years (Table 6).

\section{Discussion}

AKI is a major complication following cardiac surgery and associated with prolonged hospital stay, new-onset chronic kidney disease and increased mortality rate [2,3]. Therefore, new diagnostic criteria of CSA-AKI that could predict adverse clinical outcomes and be commonly adopted are desperately needed. The main results of the present study are that sCyC seems to be a reliable marker (1) to detect CSA-AKI and (2) combining with sCr to predict the occurrence of 3-year MAE at follow-up in patients undergoing cardiac surgery.

In the past decade there has been increasing interest in incorporating urine and serum biomarkers of AKI into clinical practice. It is anticipated that these biomarkers will enable earlier diagnosis of AKI and facilitate prognostication $[10-13,17,18]$. Among these novel 
Table 6. Predictors of MAEs at 3-year follow-up by multivariable logistic regression analysis using Firth's penalized-likelihood estimation

\begin{tabular}{llll}
\hline Covariate & OR & $95 \%$ CI & $p$ value \\
\hline CSA-AKI detected by a single marker & 3.48 & $1.27-9.58$ & 0.016 \\
CSA-AKI detected by both markers & 5.12 & $2.01-13.09$ & 0.001 \\
Age $\geq 75$ years & 2.05 & $0.89-4.73$ & 0.091 \\
Diabetes mellitus & 1.02 & $0.39-2.71$ & 0.962 \\
Recent MI & 1.20 & $0.43-3.37$ & 0.733 \\
NYHA grade III-IV & 0.90 & $0.27-3.04$ & 0.865 \\
Baseline eGFR ( $\geq 90 ; 60-89 ;<60)$ & 2.24 & $1.27-3.95$ & 0.009 \\
Cleveland Clinic Score & 1.36 & $0.87-2.13$ & 0.174 \\
\hline
\end{tabular}

CSA-AKI, cardiac surgery-associated acute kidney injury; MI, myocardial infarction; NYHA, New York Heart Association; eGFR, estimated glomerular filtration rate.

biomarkers, $\mathrm{sCyC}$ is considered the most promising functional marker for glomerular filtration $[10,17,18]$. Wan et al. [23] reported that the predictive value (the area under the ROC curve, AUC) of sCyC was 0.974, with high sensitivity and specificity, which were similar in Liu's [24], Yim's [25], and other studies. Our previous study corroborated this finding, demonstrating a significant rise in $\mathrm{sCyC}$ early after admission to the ICU following cardiac surgery in AKI patients [26]. However, with accumulating evidence, conflicting results have raised. Gaygısız et al. [27] and Martensson et al. [28] found that the predictive value (AUC) of sCyC was 0.67, with low sensitivity and specificity. In the present study, we found that a $30 \%$ increase in $\mathrm{sCyC}$ was highly accurate in diagnosing CSA-AKI, with AUC 0.843 (75\% sensitivity and $80 \%$ specificity). Then, we confirmed that patients with a 30\% increase in $\mathrm{sCyC}$ had higher Cleveland Clinic Score, which was a valid risk prediction model for severe CSA-AKI that requires dialysis. Moreover, a 30\% increase in $\mathrm{SCyC}$ was found to be an independent predictor of 3-year MAEs. Taken together, these data suggest that a 30\% increase in sCyC could be served as not only a reliable marker for ruling out CSA-AKI, but also an independent predictor of 3-year MAE.

To optimize risk stratification, we combined sCyC and sCr to create new diagnostic criteria for CSA-AKI. Among the entire cohort, 140 of CSA-AKI cases were detected by a single marker (sCyC only [ $n=95]$ vs. sCr only [ $n=45]$ ). Subgroup analysis revealed that patients in this subcohort had higher risk profiles and substantially worse long-term MAEs compared with patients without CSA-AKI. 45 patients experienced an acute rise in $\mathrm{sCr}$ without $\mathrm{SCyC}$ rise. The potential explanations are unknown and the case volume prevented us from performing further analysis. Future study is needed to address the issue whether the difference exists between the patients with an acute rise in $\mathrm{sCyC}$ only and patients with an acute rise in $\mathrm{sCr}$ only. Another 133 CSA-AKI cases were detected by both sCyC- and sCr-based criteria. A significant increase in risk of long-term MAEs was observed in this group of patients. Multivariable logistic regression analysis showed that the predictability of MAEs increased stepwise across the 3 groups. Consistent with the results of previous studies, our data indicated that $\mathrm{sCyC}$ is a more sensitive marker than $\mathrm{sCr}$ in identifying CSA-AKI cases. The reason might be that $\mathrm{sCyC}$ is thought to be less influenced by non-glomerular filtration rate determinants, is extracellular, and has a smaller volume of distribution compared with creatinine [14-16]; it may be a better measure of AKI than creatinine in the perioperative period [24, 26]. Patients with both sCyC- and sCr-based CSA-AKI had the poorest long-term outcomes, implying that inclusion of both $\mathrm{sCyC}$ and $\mathrm{sCr}$ in defining CSA-AKI would increase the association between CSA-AKI and clinical outcomes. Equations to estimate GFR based on both $\mathrm{sCyC}$ and $\mathrm{sCr}$ have been developed [29]; combining sCyC and sCr to define CSA-AKI might be applied to clinical 
practice in the future. In our center, testing of $\mathrm{sCyC}$ is readily accessible to most practitioners, which therefore is part of routine clinical practice. Preoperative renal dysfunction was identified as an important risk factor for the development of AKI [21]. Our data suggests that it may be more important than other risk factors for long-term MAEs, which go beyond circular reasoning.

Several limitations of the present study should be acknowledged. First, our data is derived from a single center study. The result of our data should be confirmed by a further larger multicenter study. Second, as we used unselected material we are unable to make any statement about the value of the new definition of CSA-AKI in specific subpopulations and circumstances. Third, other urine markers of CSA-AKI such as neutrophil gelatinase-associated lipocalin and kidney injury molecule 1 were not routinely measured. Data regarding the association between the new definition of CSA-AKI and renal injury markers were unavailable in the present study. Finally, only one new onset of end-stage renal disease requiring chronic dialysis was observed in the present cohort. The correlation between new AKI definition and adverse renal outcomes should be further assessed.

In conclusion, measurement of $\mathrm{sCyC}$ might be adopted not only as a diagnostic test for AKI but, more importantly, as a prognostic tool for future MAE among patients undergoing cardiac surgery. Moreover, abnormal values of both markers can potentially identify the highest-risk subset of CSA-AKI patients, in whom careful monitoring for adverse events is required.

\section{Acknowledgment}

The present study was supported by the Shanghai Medical Development Grant (2003ZD001) and the National Natural Science Foundation of China (No. 81170687).

\section{Statement of Ethics}

The study protocol was approved by the Institutional Research Ethics Board. Informed consent was obtained from all participants.

\section{Disclosure Statement}

None of the other authors have any conflict of interest to declare.

\section{References}

1 Bove T, Monaco F, Covello RD, Zangrillo A. Acute renal failure and cardiac surgery. HSR Proc Intensive Care Cardiovasc Anesth. 2009;1(3):13-21.

2 Dasta JF, Kane-Gill SL, Durtschi AJ, Pathak DS, Kellum JA. Costs and outcomes of acute kidney injury (AKI) following cardiac surgery. Nephrol Dial Transplant. 2008 Jun;23(6):1970-4.

3 Wang Y, Bellomo R. Cardiac surgery-associated acute kidney injury: risk factors, pathophysiology and treatment. Nat Rev Nephrol. 2017 Nov;13(11):697-711.

4 Howell NJ, Freemantle N, Bonser RS, Graham TR, Mascaro J, Rooney SJ, et al. Subtle changes in renal function are associated with differences in late survival following adult cardiac surgery. Eur J Cardiothorac Surg. 2012 Apr;41(4):e38-42.

5 Englberger L, Suri RM, Li Z, Casey ET, Daly RC, Dearani JA, et al. Clinical accuracy of RIFLE and Acute Kidney Injury Network (AKIN) criteria for acute kidney injury in patients undergoing cardiac surgery. Crit Care. 2011; 15(1):R16. 


\section{Kidney \\ Blood Pressure \\ Research}

\begin{tabular}{l|l}
\hline Kidney Blood Press Res 2019;44:415-425 \\
\hline DOI: 10.1159/000499647 & $\begin{array}{l}\text { @ 2019 The Author(s). Published by S. Karger AG, Basel } \\
\text { www.karger.com/kbr }\end{array}$ \\
\hline
\end{tabular}

Che et al.: Use of Both sCyC and sCr as Diagnostic Criteria for CSA-AKI

6 Mao H, Katz N, Ariyanon W, Blanca-Martos L, Adýbelli Z, Giuliani A, et al. Cardiac surgery-associated acute kidney injury. Blood Purif. 2014;37 Suppl 2:34-50.

7 Swaminathan M, Phillips-Bute BG, Conlon PJ, Smith PK, Newman MF, Stafford-Smith M. The association of lowest hematocrit during cardiopulmonary bypass with acute renal injury after coronary artery bypass surgery. Ann Thorac Surg. 2003 Sep;76(3):784-91.

8 Lassnigg A, Schmidlin D, Mouhieddine M, Bachmann LM, Druml W, Bauer P, et al. Minimal changes of serum creatinine predict prognosis in patients after cardiothoracic surgery: a prospective cohort study. J Am Soc Nephrol. 2004 Jun;15(6):1597-605.

9 Levey AS, Bosch JP, Lewis JB, Greene T, Rogers N, Roth D; Modification of Diet in Renal Disease Study Group. A more accurate method to estimate glomerular filtration rate from serum creatinine: a new prediction equation. Ann Intern Med. 1999 Mar;130(6):461-70.

10 Wald R, Liangos O, Perianayagam MC, Kolyada A, Herget-Rosenthal S, Mazer CD, et al. Plasma cystatin C and acute kidney injury after cardiopulmonary bypass. Clin J Am Soc Nephrol. 2010 Aug;5(8):1373-9.

11 Srisawat N, Praditpornsilpa K, Patarakul K, Techapornrung M, Daraswang T, Sukmark T, et al.; Thai Lepto-AKI study group. Neutrophil gelatinase associated lipocalin (NGAL) in leptospirosis acute kidney injury: a multicenter study in thailand. PLoS One. 2015 Dec;10(12):e0143367.

12 Nisula S, Yang R, Poukkanen M, Vaara ST, Kaukonen KM, Tallgren M, et al.; FINNAKI Study Group. Predictive value of urine interleukin-18 in the evolution and outcome of acute kidney injury in critically ill adult patients. Br J Anaesth. 2015 Mar;114(3):460-8.

13 Parikh CR, Thiessen-Philbrook H, Garg AX, Kadiyala D, Shlipak MG, Koyner JL, et al.; TRIBE-AKI Consortium. Performance of kidney injury molecule- 1 and liver fatty acid-binding protein and combined biomarkers of AKI after cardiac surgery. Clin J Am Soc Nephrol. 2013 Jul;8(7):1079-88.

14 Dharnidharka VR, Kwon C, Stevens G. Serum cystatin C is superior to serum creatinine as a marker of kidney function: a meta-analysis. Am J Kidney Dis. 2002 Aug;40(2):221-6.

15 Odutayo A, Cherney D. Cystatin C and acute changes in glomerular filtration rate. Clin Nephrol. 2012 Jul;78(1): 64-75.

16 Sjöström P, Tidman M, Jones I. The shorter T1/2 of cystatin C explains the earlier change of its serum level compared to serum creatinine. Clin Nephrol. 2004 Sep;62(3):241-2.

17 Yong Z, Pei X, Zhu B, Yuan H, Zhao W: Predictive value of serum cystatin C for acute kidney injury in adults: a meta-analysis of prospective cohort trials. Sci Rep. 2017 Jan 23;7:41012.

18 Nejat M, Pickering JW, Walker RJ, Endre ZH. Rapid detection of acute kidney injury by plasma cystatin C in the intensive care unit. Nephrol Dial Transplant. 2010 0ct;25(10):3283-9.

19 Coca SG, Yalavarthy R, Concato J, Parikh CR. Biomarkers for the diagnosis and risk stratification of acute kidney injury: a systematic review. Kidney Int. 2008 May;73(9):1008-16.

20 KDIGO. Clinical practice guideline for acute kidney injury section 2: AKI definition. Kidney Int Suppl. 2012;2: 19-36.

21 Thakar CV, Arrigain S, Worley S, Yared JP, Paganini EP. A clinical score to predict acute renal failure after cardiac surgery. J Am Soc Nephrol. 2005 Jan;16(1):162-8.

22 Firth D. Bias reduction of maximum likelihood estimates. Biometrika. 1993;80(1):27-38.

23 Wan ZH, Wang JJ, You SL, Liu HL, Zhu B, Zang H, et al. Cystatin C is a biomarker for predicting acute kidney injury in patients with acute-on-chronic liver failure. World J Gastroenterol. 2013 Dec;19(48):9432-8.

24 Liu YJ, Sun HD, Chen J, Chen MY, Ouyang B, Guan XD. Klotho: a novel and early biomarker of acute kidney injury after cardiac valve replacement surgery in adults. Int J Clin Exp Med. 2015 May;8(5):7351-8.

25 Yim H, Kym D, Seo DK, Yoon J, Yang HT, Lee J, et al. Serum cystatin C and microalbuminuria in burn patients with acute kidney injury. Eur J Clin Invest. 2015 Jun;45(6):594-600.

26 Che M, Xie B, Xue S, Dai H, Qian J, Ni Z, et al. Clinical usefulness of novel biomarkers for the detection of acute kidney injury following elective cardiac surgery. Nephron Clin Pract. 2010;115(1):c66-72.

27 Gaygısız Ü, Aydoğdu M, Badoğlu M, Boyacı N, Güllü Z, Gürsel G. Can admission serum cystatin C level be an early marker subclinical acute kidney injury in critical care patients? Scand J Clin Lab Invest. 2016;76(2): 143-50.

28 Mårtensson J, Jonsson N, Glassford NJ, Bell M, Martling CR, Bellomo R, et al. Plasma endostatin may improve acute kidney injury risk prediction in critically ill patients. Ann Intensive Care. 2016 Dec;6(1):6.

29 Zappitelli M, Parvex P, Joseph L, Paradis G, Grey V, Lau S, et al. Derivation and validation of cystatin C-based prediction equations for GFR in children. Am J Kidney Dis. 2006 Aug;48(2):221-30. 